\title{
Adaptive Error-Resilience Transcoding and Fairness Grouping for Video Multicast over Wireless Networks
}

\author{
Chih-Ming Chen ${ }^{1,3}$, Chia-Wen Lin $^{2}$ and Yung-Chang Chen ${ }^{1}$ \\ ${ }^{1}$ Department of Electrical Engineering, National Tsing Hua University, \\ Hsinchu, Taiwan 30013, R.O.C., ycchen@ee.nthu.edu.tw \\ ${ }^{2}$ Department of Computer Science \& Information Engineering, National Chung Cheng University, \\ Chiayi, Taiwan 621, R.O.C., cwlin@cs.ccu.edu.tw \\ ${ }^{3}$ Chunghwa Telecom Co., Ltd.
}

\begin{abstract}
In this paper, we present a two-pass intra-refresh transcoder for on-the-fly enhancing error resilience of a compressed video in a three-tier streaming system. Furthermore, we consider the problem of multicasting a video to multiple clients with diverse channel conditions. We propose a MINMAX loss rate estimation scheme to determine a single intrarefresh rate for all the clients in a multicast group. For the scenario that a quality variation constraint is imposed on the users, we also propose a grouping method to partition a multicast group of heterogeneous users into a minimal number of sub-groups to minimize the channel bandwidth consumption while meeting the quality variation constraint and achieving fairness among all sub-groups. Experimental results show that the proposed method can effectively mitigate the error propagation due to packet loss as well as achieve fairness not only among all sub-groups and also clients in a multicast group.
\end{abstract}

\section{INTRODUCTION}

Transmitting video data over error prone networks can be very unreliable due to packet loss, and still present many challenges to streaming video applications, especially for mobile video. In a non-live video streaming system, a server stores pre-encoded video bitstreams and transmits them to client terminals for decoding and playback. The existing video encoding techniques exploit spatial and temporal redundancy to achieve a high compression ratio, while making the compressed data very sensitive to transmission error. The packet loss problem may lead to serious video quality degradation, which not only affects the quality of a corrupted frame, but also leads to error propagation to its subsequent frames due to the motion-compensated prediction technique used in current standard video codecs. In order to achieve error robustness for transmitting video over wireless networks, the server located in an intermediate network node must be able to adapt or transcode the non-error-resilient compressed video bitstreams into error-resilience-capable bitstreams. To serve this purpose, a video transcoder can be placed in a network node (e.g., a mobile switch/base-station, a proxy server, or a video gateway) connected to a high-loss network (e.g., wireless network or highly congested network) to insert error resilience features into the video bitstream to achieve robust video transmission over wireless channels [1]-[5].

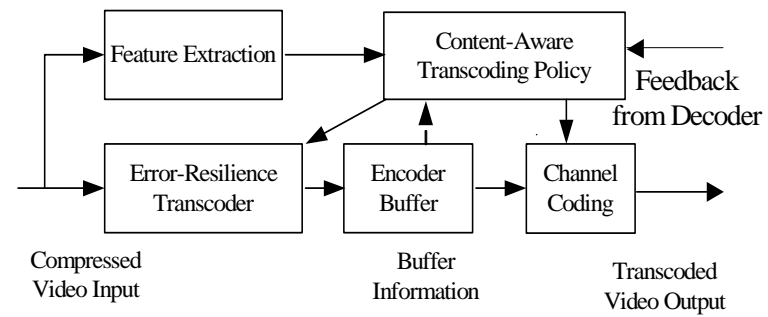

Fig. 1. Proposed system framework of error-resilience video transcoder.

A three-tier streaming system, using a transcoder to handle the different demands (e.g., bandwidth, resolution, frame-rate, and channel condition) from different client devices can reduce the complexity and transmission cost from the streaming server to the home receivers. Fig. 1 shows the proposed error resilience transcoder with feedback channels. The transcoder first extracts the video features (e.g., locations of video data which are likely to result in more serious error propagation if lost) from the incoming bitstream as well as estimates the client channel conditions according to the feedback channel statistics. The extracted features and the estimated channel condition are then used to determine the error resilience transcoding policy that guides the allocation of source and channel coding resources. In our previous work [4][5], a novel two-pass error resilience transcoder using content-aware intra-refresh with profit tracing (CAIR-PT) was proposed. Adopting intra-refresh as an error resilience coding tool [6]-[8] avoids making any change for standard video decoders, which is important in terms of cost and convenience for many practical applications. 
The problem of multicasting a video to multiple clients with diverse channel conditions is considered in this paper. Ammar et al. developed a destination set grouping (DSG) protocol to improve inter-receiver fairness [9] for multicast communication. They defined a single receiver fairness fuction that maps from the actual operating rate to a fairness value of users. However, the fairness function is in general application dependent. In order to constrain the quality variation for a group of heterogeneous receivers, a weighted average criterion was proposed in [10] that maximizes the individual performance over the users with reasonable channel conditions and ignores the users that have channel conditions with higher packet loss rate than a preset threshold. However, how to select an applicable threshold to balance the fairness and the visual quality of users with various channel conditions is another problem.

In this paper, we propose an efficient method to cope with more general video multicasting situations involving heterogeneous clients with diverse channel conditions. We present a MINMAX loss rate estimation scheme to determine an appropriate intra-refresh rate for all the clients in a multicast group. We also propose a grouping method to partition a group of heterogeneous users into a minimal number of sub-groups to meet a given quality variation constraint while minimizing the channel bandwidth consumption under the quality constraint as well as achieving fairness among all sub-groups.

The remainder of this paper is organized as follows. The proposed two-pass error-resilient transcoding scheme using prioritized intra-refresh with profit tracing is presented in Section II. A MINMAX intra-refresh rate allocation strategy for video multicast in heterogeneous environments is proposed in Section III. A grouping strategy for divide a multicast group into smaller subgroups to meet the quality variation constraint is also presented. Section IV shows experimental results. Finally the conclusions are drawn in Section V.

\section{ERROR-RESILIENCE TRANSCODING USING PRIORITIZED INTRA-REFRESH}

We summarize our CAIR-PT error resilience transcoder proposed in [4][5] in this section. In the proposed two-pass CAIR-PT transcoder, the front-end encoder utilizes the motion vectors generated in the encoding process and the estimated concealment distortion to evaluate the error propagation effect at the macroblock and frame levels within a group of pictures (GOP) as the side information which is stored in the streaming server to be used as transcoding hints for guiding intra-refresh allocation. In the transcoding process, the transcoder uses the side information received from the streaming server and the client acket loss rates collected from feedback channels to determine an intra-refresh allocation for each frame of a GOP, and then performs intra-refresh on a number of high-priority macroblocks with highest lossimpact factors according to the intra-refresh allocation. The key idea behind the proposed transcoding scheme is to stop the error propagation in the current frame by performing intra-refresh on those macroblocks which reference high loss-impact prediction blocks of the previous frame.

We define a pixel-level loss-impact (LI) metric as the product of two parameters: PRC (Pixel Reference Count) and PCE (Pixel Concealment Error), to characterize the amount of pixel-wise error propagation as follows:

$$
L I(x, y, n)=\operatorname{PCE}(x, y, n) \cdot \operatorname{PRC}(x, y, n)
$$

where $P R C(x, y, n)$ represents the frequency of pixel $(x, y)$ of frame $n$ being referenced by pixels in the succeeding frames within a GOP in the motion-compensated prediction process. It can be calculated recursively by summing up the individual reference counts of pixels in frame $n+1$ which reference the pixel $(x, y)$ of frame $n$ in the reverse tracking order from the last frame to the first frame of a GOP as follows:

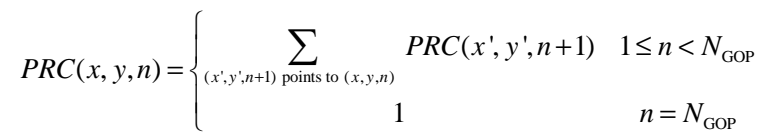

$\operatorname{PCE}(x, y, n)$, as defined in (3), denotes the norm of concealment error of pixel $(x, y)$ of frame $n$ should this pixel be corrupted.

$$
\operatorname{PCE}(x, y, n)=|f(x, y, n)-f(x, y, n-1)|^{2}
$$

where $f(x, y, n)$ represents the pixel value of pixel $(x, y)$ in frame $n$. In this work, the zero-motion error concealment scheme was adopted to compute the concealment error.

We then use the motion information to calculate the current frame's macroblock-level error-propagation (from the previous frames) as follows:

$$
E P_{\mathrm{MB}}(m, n)=\sum_{(x, y) \in \mathrm{MB}_{m}} L I\left(x+M V_{x}, y+M V_{y}, n-1\right)
$$

where $m$ denotes the macroblock index in a frame, and $\left(M V_{\mathrm{x}}, M V_{\mathrm{y}}\right)$ is the associated motion vector of pixel $(x, y)$.

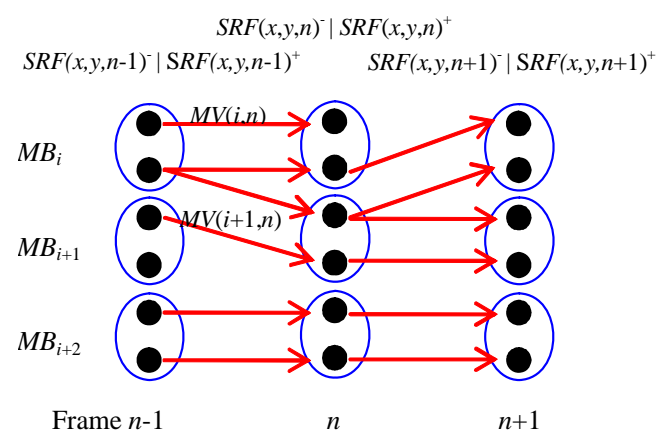

Fig. 2. Profit tracing of each refreshed macroblock. 
A pixel-wise surplus refresh factor (SRF) is also defined, which is inherited from a previous intra-coded macroblock. As illustrated in Fig. 2, $\operatorname{SRF}(x, y, n)^{-}$represents the intermediate $S R F$ of pixel $(x, y)$ in frame $n$ before the transcoder decides the coding mode of the macroblock containing the pixel, as defined in (5).

$$
\operatorname{SRF}(x, y, n)^{-}=\operatorname{SRF}\left(x+M V_{x}, y+M V_{y}, n-1\right)^{+} \cdot(1-P L R)
$$

In (5), for the sake of simplicity, we use the packet loss rate $P L R$ to approximate the pixel loss rate in a GOP, since the two loss rates usually have close values for a sufficiently large amount of data (e.g., a GOP). $\operatorname{SRF}(x, y, n)^{+}$is set to be 1 , if pixel $(x, y)$ belongs to an intrarefreshed macroblock. Otherwise, $\operatorname{SRF}(x, y, n)^{+}$remains the same as $\operatorname{SRF}(x, y, n)^{-}$. The initial values, $\operatorname{SRF}(x, y, 0)^{-}$, are all set to 0 . Besides, in an initial I-frame, the values of $\operatorname{SRF}(x, y, 0)^{+}$are all set to 1 , as summarized in (6).

$$
\left\{\begin{array}{cc}
\operatorname{SRF}(x, y, n)^{+}=1, & (x, y) \in \text { intra-MB } \\
\operatorname{SRF}(x, y, n)^{+}=\operatorname{SRF}(x, y, n)^{-}, & (x, y) \in \text { inter-MB } \\
\operatorname{SRF}(x, y, 0)^{-}=0 & \\
\operatorname{SRF}(x, y, 0)^{+}=1 &
\end{array}\right.
$$

We use the motion information to map pixel-level $\operatorname{SRF}(x, y, n-1)^{+}$from the previous frame to obtain the macroblock-level $\overline{\operatorname{SRF}}_{\mathrm{MB}}(m, n)$ with a value of ranging from 0 to 1 , as follows:

$$
\overline{\operatorname{SRF}}_{\mathrm{MB}}(m, n)=\frac{1}{\operatorname{SIZE} E_{\mathrm{MB}}} \sum_{(x, y) \in M B_{m}} \operatorname{SRF}(x, y, n)^{-}
$$

where $S I Z E_{\mathrm{MB}}$ represents the number of pixels in a macroblock. After computing $\overline{\operatorname{SRF}}_{\mathrm{MB}}(m, n)$, we select a total of $N_{\text {intra }}(n)$ macroblocks with top-ranking $E P_{\mathrm{MB}}(m, n) \cdot\left\{1-\overline{S R F}_{\mathrm{MB}}(m, n)\right\}$ values to perform intrarefresh for the $n$-th frame of a GOP.

Finally, all $E P_{\mathrm{MB}}$ 's in each frame are summed up to estimate the frame-level error-propagation as follows:

$$
E P_{n}=\sum_{m=1}^{N_{\mathrm{MB}}^{\mathrm{F}}} E P_{\mathrm{MB}}(m, n)
$$

where $N_{\mathrm{MB}}^{\mathrm{F}}$ denotes the number of macroblocks in a frame.

In the second-pass transcoding, the intra-refresh rate and the intra-block allocation strategy for each GOP are determined so as to adapt the transcoded video to varying network conditions. One key issue of the intra-refresh algorithm is to determine the number of macroblocks to be intra-coded in a GOP.

$$
N_{\text {intra }}^{\mathrm{GOP}}=\frac{\frac{1}{N_{\mathrm{GOP}}} \sum_{n=2}^{N_{\mathrm{GOP}}} E P_{n} \cdot P L R_{\mathrm{TC}}}{T H_{\text {intra }}}
$$

where $N_{\text {intra }}^{\mathrm{GOP}}$ represents the total number of macroblocks of P-frames to be intra-refreshed in a GOP, $N_{\mathrm{GOP}}$ denotes the GOP size, $P L R_{\mathrm{TC}}$ represents the channel packet loss rate estimated at the transcoder by using the client feedback information and is updated every GOP to capture frequently changing network conditions, and $T H_{\text {intra }}$ is a scaling parameter.

The intra-refresh allocation is then distributed to a GOP using the following algorithm:

If $n=2$ (i.e., the first P-frame in a GOP)

$$
N_{\text {intra }}(n)=\frac{E P_{n}}{\sum_{i=n}^{N_{\text {GOP }}} E P_{i}} \cdot N_{\text {intra }}^{\mathrm{GOP}}
$$

else if $3 \leq n \leq N_{\mathrm{GOP}}$

$$
N_{\text {intra }}(n)=\frac{E P_{n}}{\sum_{i=n}^{N_{\text {GOP }}} E P_{i}} \cdot\left(N_{\text {intra }}^{\mathrm{GOP}}-\sum_{i=2}^{n-1} N_{\text {intra }}(i)\right)
$$

\section{end if}

where $N_{\text {intra }}(n)$ denotes the number of macroblocks with top-ranking $E P_{\mathrm{MB}}$ values to be intra-coded in frame $n$.

\section{INTRA-REFRESH STRATEGY FOR VIDEO MULTICAST}

In many practical applications, the bitstream may need to be simultaneously delivered to multiple clients with diverse channel characteristics. How to determine in the transcoder an appropriate parameter, $P L R_{\mathrm{TC}}$, for a single multicast stream delivered to multiple clients with different channel loss characteristics, $P L R_{\mathrm{ch}}$ 's, is a practical problem in video multicast applications. The optimal intra-refresh rate allocation for video multicasting is still an open problem, which, to our best knowledge, has not yet been well addressed. Moreover, should a constraint on quality variation be imposed for mobile clients with heterogeneous channel characteristics, sending a single bitstream may not be able to meet the constraint. How to partition the clients in a multicast group into a minimal number of sub-groups so as to minimize the required channel bandwidth while meeting the quality variation constraint for the clients is also of interest.

\section{A. MINMAX penalty criterion}

To characterize the amount of quality penalty of adopting at the transcoder an estimated packet loss rate, $P L R_{\mathrm{TC}}$, which does not exactly match the packet loss rate of a channel, $P L R_{\mathrm{ch}}$, we define the following PSNR penalty metric:

$\Delta P S N R_{i}\left(x \mid P_{i}\right)=\operatorname{PSNR}\left(P L R_{\mathrm{TC}}=x \mid P L R_{\mathrm{ch}}=p_{i}\right)-\operatorname{PSNR}\left(P L R_{\mathrm{TC}}=p_{i} \mid P L R_{\mathrm{ch}}=p_{i}\right)$ 
where we assume the packet loss rate of the $i$-th client is $p_{i}$, whereas the transcoder uses a different $P L R_{\mathrm{TC}}=x$ to determine the intra-refresh rate for the outgoing video bitstream using (11). If the transcoder adopts a $P L R_{\mathrm{TC}}$ different from the optimal value (i.e., the channel packet loss rate $\left.P L R_{\mathrm{ch}}\right)$, a $P L R_{\mathrm{TC}}$ value smaller than $P L R_{\mathrm{ch}}$ will lead to more severe error propagation caused by packet loss, since the intra-refresh rate is not sufficient to stop the error propagation effectively. On the other hand, a $P L R_{\mathrm{TC}}$ value higher than $P L R_{\mathrm{ch}}$ will lead to an excessive intrarefresh rate, resulting in poor coding efficiency which cannot be well compensated for by the performance gain obtained from the enhanced error resiliency.

When multicasting a video bitstream to multiple clients with diverse loss characteristics, the transcoder should not just maximize the received visual quality for some client since it may lead to quality degradation for the others. We propose to determine $P L R_{\mathrm{TC}}$ based on the following MINMAX penalty criterion:

$$
P L R_{\mathrm{TC}}^{\mathrm{opt}}=\arg \min _{x} \max _{i}\left\{\left|\Delta P S N R_{i}\left(x \mid p_{i}\right)\right|\right\}
$$

The transcoder then uses $P L R_{\mathrm{TC}}^{\mathrm{opt}}$ to determine the intra-refresh rate for the outgoing video bitstream according to (11). Such a single intra-refresh rate will result in quality penalty $\triangle P S N R_{i}\left(x \mid p_{i}\right)$ for the $i$-th channel due to the mismatch of channel-loss rates between $P L R_{\mathrm{TC}}$ and $p_{i}$. The intra-refresh rate $P L R_{\mathrm{TC}}^{\mathrm{opt}}$ is optimal for a multicast group in the sense of minimizing the maximum penalty distortion that any client will suffer, thereby tending to reduce the distortion deviation among all clients to achieve fairness.

In order to obtain $P L R_{\mathrm{TC}}^{\mathrm{opt}}$ analytically, we propose the following model to characterize the channel mismatch distortion:

$$
\Delta P S N R(x \mid p)=\left\{\begin{array}{cc}
G_{0} \cdot(p-x) \cdot e^{-m \cdot x} & x<p \\
G_{1} \cdot(x-p) \cdot e^{n \cdot(x-100)} & x \geq p
\end{array}\right.
$$

where $G_{i}=c_{i}-k_{i} \times p^{1 / 3}$.

As mentioned above, the penalty, ${ }_{P S N R}(x \mid p)$, is mainly caused by error propagation when $x<p$, and by coding efficiency loss when $x \geq p$. They depend on the diverged distance of $x$ from $p$. The parameters $G_{0}$ and $G_{1}$ are decreased by a scale $k_{i}$ from $c_{i}$ to indicate the slope of decay. $e^{-m \cdot x}$ and $e^{n \cdot(x-100)}$ are used to fine tune the smoothness. We use a fixed set of parameters, which can be computed beforehand and stored as side information, to model each individual video bitstream. For example, the set of model parameters used for Foreman is $\left(c_{0}, c_{1}, k_{0}, k_{1}\right.$, $m, n)=(0.53,3.29,0.01,1.15,0.35,0.035)$, and Fig. 3 illustrates the penalty models for the Salesman and
Carphone sequence under three channel packet loss rates: $P L R_{\mathrm{ch}}=5 \%, 10 \%$, and $15 \%$, respectively.
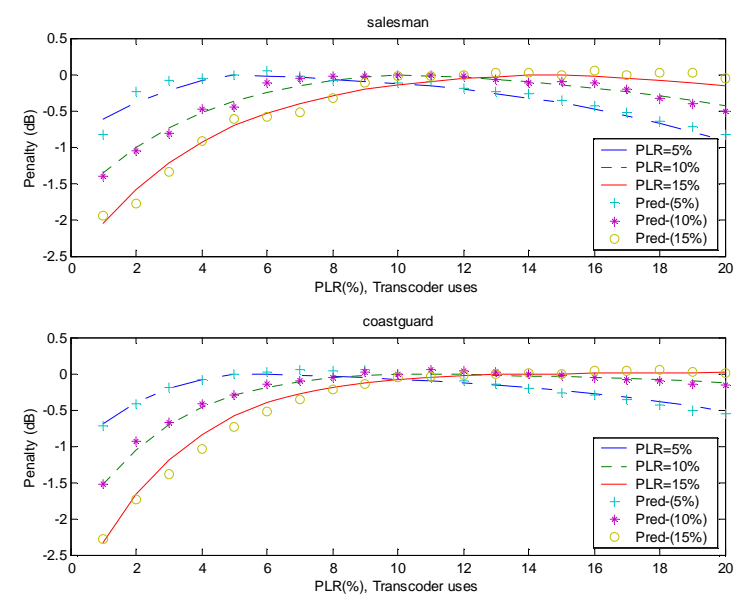

Fig. 3. Plots of PNSR penalty caused by using at the transcoder an estimated packet-loss rate that mismatches the packet-loss rates of individual channels for Salesman and Carphone.

\section{B. Fairness grouping}

A video multicast session may involve a large number of receivers with heterogeneous channel conditions. This usually leads to a tradeoff between bandwidth utilization efficiency and granularity of error control. On one hand, sending a single video bitstream to all receivers in the multicast group achieves the best bandwidth utilization efficiency, but leads to the coarsest granularity of error control. On the other hand, sending an individual bitstream to each receiver leads to the finest granularity of error control but the worst bandwidth utilization efficiency. Considering the fairness among the receivers in a multicast group, it is usually undesirable to trade the visual quality of users with good channel conditions for the visual quality of users with significantly poor channel conditions, especially in WLAN environments where client mobility may temporarily result in rather unstable transient channel behaviors. In order to constrain the quality variation for a group of heterogeneous receivers, we propose to take into account the heterogeneity of the receivers' channel conditions to decide whether to divide the receivers with temporarily unstable or relatively diverse channel characteristics into subgroups and then send video bitstreams of different intra-refresh rates to individual subgroups according to a MINMAX criterion. Based on the proposed penalty model, we attempt to partition receivers in a multicast group into a minimal number of sub-groups so as to minimize the required channel bandwidth while meeting the quality variation constraint for each subgroup as well as achieving fairness among all sub-groups.

As shown in Fig. 4, the MINMAX point for a multicast group will stay at the cross-point of the two 
penalty model curves with the lowest and highest PLRs. Suppose there exist $N$ receivers in the multicast group with $K$ classes of packet loss rates, $\left\{P L R_{1}, P L R_{2} \ldots, P L R_{i} \ldots\right.$, $\left.P L R_{K}\right\}$, where $P L R_{i-1}<P L R_{i}$ and $K \leq N$. In Fig. 4, $D_{i, j}$ denotes the quality penalty value at the cross-point of the penalty model curves with the two packet loss rates: $P L R_{i}$ and $P L R_{j}$. Note that, $D_{i, i}=0$ and $D_{i, j}=D_{j, i}$. In our grouping strategy, as shown in Fig. 5, a cross-point matrix is used to record the PSNR penalty values of cross-points of every two penalty model curves. If the receivers with $P L R_{i}$, $P L R_{i+1} \ldots$, and $P L R_{j}$ are grouped together as one sub-group, the penalty value for the subgroup becomes the MINMAX penalty value of the subgroup (i.e., $D_{i, j}$ ). Considering the efficiency of bandwidth utilization, our goal is to partition the $K$ classes of PLRs into a minimal number of subgroups $L(1 \leq L \leq K)$ so as to maximize the channel utilization efficiency while meeting the constraint of quality variation $\left(Q V_{\max }\right)$ for each sub-group.

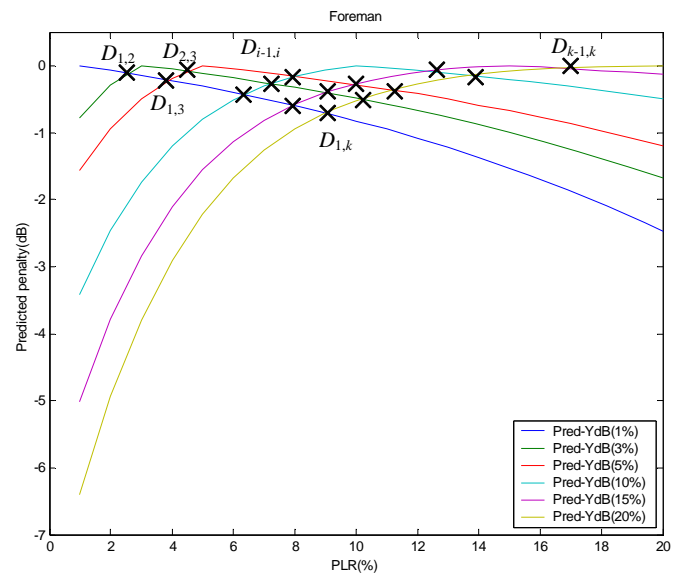

Fig. 4. Cross points of the predicted PSNR penalty of any two receivers with different $P L R$ s.

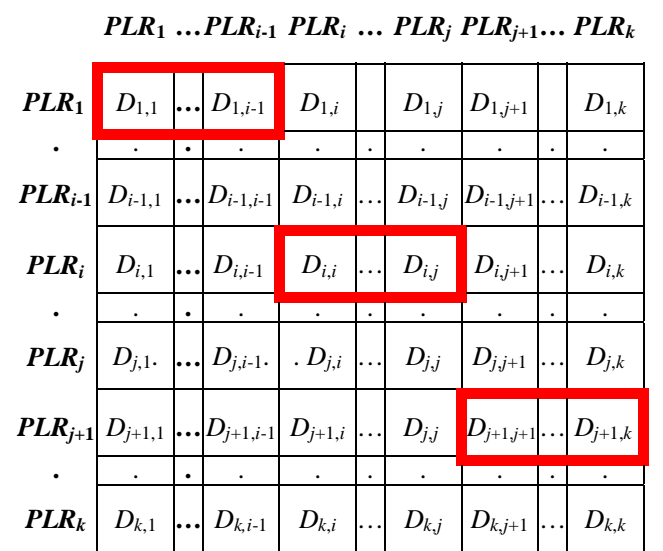

Fig. 5. A cross-point matrix records the penalty distortion values of each cross point.
In order to achieve fairness among all sub-groups, we propose to minimize the maximal quality penalty value of the sub-groups under the constraint of quality variation $\left(Q V_{\max }\right)$ as follows:

$$
\begin{aligned}
Q V_{\text {min max }}= & \min _{\left[i_{m-1}+1, i_{m}\right] \in m \text {-th group }} \max _{m}\left\{D_{i_{m-1}+1, i_{m}}\right\} \text { for } m=1, \ldots, L \\
& \text { subject to } Q V_{\text {min max }}<Q V_{\text {max }}
\end{aligned}
$$

where $L$ represents the number of subgroups that the $N$ receivers with $K$ classes of packet loss rates will be partitioned into. $Q V_{\min \max }$ represents the MINMAX quality penalty value of the $L$ subgroups. In order to maximize the channel utilization efficiency, we would like to minimize the number of subgroups, while meeting the constraint that $Q V_{\min \max }$ must be less than $Q V_{\max }$.

\section{EXPERIMENTAL RESULTS}

In our experiments, three QCIF (176x144) test sequences are pre-encoded at $30 \mathrm{fps}$ and $384 \mathrm{Kbps}$ with the IPPP...GOP structure with a GOP size of 30 . We implemented a cascaded pixel-domain transcoder [11] using an MPEG-4 public-domain software codec [12] to perform the adaptive intra-refresh transcoding. The output bit-rate, after inserting intra-refresh macroblocks, is regulated to the same bit-rate of the input video (i.e., 384 kbps) by using the MPEG-4 TM5 rate control scheme. In our experiments, a slice which contains one row of macroblocks is encapsulated into one packet. In this work, we adopt a simplified Gilbert channel at the packet level [13] to generate packet loss patterns for simulation. The average burst length is set to one to simulate random packet loss situations as in fast-fading channels. For slowfading channels which will result in longer burst losses, packet interleaving techniques can be used to spread a burst loss into individual single-packet losses to facilitate the error control process if the introduced complexity and delay are acceptable.

A. Performance of multicast with one single bitstream

We apply the penalty model functions in (14) to compute the optimal $P L R_{\mathrm{TC}}$ which meets the MINMAX criterion for the application scenario involving six receivers with different channel loss rates as listed in Table 1. Table 3 also shows the numerical results of the penalty distortion $\triangle P S N R_{i}\left(x \mid p_{i}\right)$ for each user with channel loss rate $\mathrm{p}_{\mathrm{i}}$, where 'Average' refers to $x=\left(\sum_{i=1}^{6} p_{i}\right) / 6$, and $x=\max \left\{p_{i}\right\}$ for 'Worst,' whereas $x=\min \left\{p_{i}\right\}$ for 'Best.' The results show that the proposed MINMAX penalty criterion yields the best visual quality in terms of the mean and the variance of PSNR penalty values among the four methods. 
Table 1. Comparison of penalty in distortion for six users under different case

\begin{tabular}{|c|c|c|c|c|c|}
\hline User & $p_{i}(\%)$ & MINMAX & Average & Worst & Best \\
\hline 1 & 3 & 0.03 & 0.01 & 0.54 & 0 \\
\hline 2 & 3 & 0.01 & 0.06 & 0.36 & 0 \\
\hline 3 & 3 & 0.05 & 0.03 & 0.60 & 0 \\
\hline 4 & 5 & 0.03 & 0 & 0.22 & 0.34 \\
\hline 5 & 5 & 0.01 & 0 & 0.28 & 0.43 \\
\hline 6 & 10 & 0.24 & 0.45 & 0 & 1.07 \\
\hline
\end{tabular}

B. Performance of multicast with multiple bitstreams

For the multiple steams experiment, 13 users with heterogeneous PLRs, $\{1 \%, 1 \%, 1 \%, 1 \%, 1 \%, 3 \%, 3 \%, 3 \%$, $5 \%, 5 \%, 10 \%, 15 \%, 20 \%$, are considered in the multicast scenario. Suppose the constraint of quality variation $\left(Q V_{\max }\right)$ for each sub-group is $0.5 \mathrm{~dB}$. In the case of sending only a single bitstream to all clients, the MINMAX penalty distortion value is $D_{16}=0.71 \mathrm{~dB}$ that exceeds the constraint of $Q V_{\max }$. The resulting MINMAX quality penalty value $Q V_{\min \max }$, which is the maximal value of MINMAX penalty distortion values of Subgroup $\# 1\left(D_{13}=0.21 \mathrm{~dB}\right)$ and Subgroup \#2 $\left(D_{46}=0.15 \mathrm{~dB}\right)$, is $0.21 \mathrm{~dB}$ which can meet the constraint of $Q V_{\text {max. }}$ The result indicates that, in this case, partitioning the clients into two sub-groups and sending two bitstreams for individual subgroups accordingly can achieve the best bandwidth utilization efficiency while meeting the constraint of $Q V_{\max }$. The difference of the maximal penalty distortion of Subgroup \#1 and \#2 is $0.06 \mathrm{~dB}$ which is the minimum value as the clients are partitioned into two sub-groups. Therefore, fairness can be achieved among all sub-groups. As shown in Table 2, while sending two bitstreams, the fourth user with $1 \%$ PLR has the largest PSNR penalty of $0.17 \mathrm{~dB}$ in Group \#1 and the thirteenth user with $20 \%$ $P L R$ has $0.1 \mathrm{~dB}$ penalty in Group \#2, leading to $0.83 \mathrm{~dB}$ improvement compared to sending only one single bitstream that results in a largest penalty of $1 \mathrm{~dB}$. Sending two bitstreams, however, will double the bandwidth required.

Table 2. Penalty in distortion for fourteen users for one single and two streams

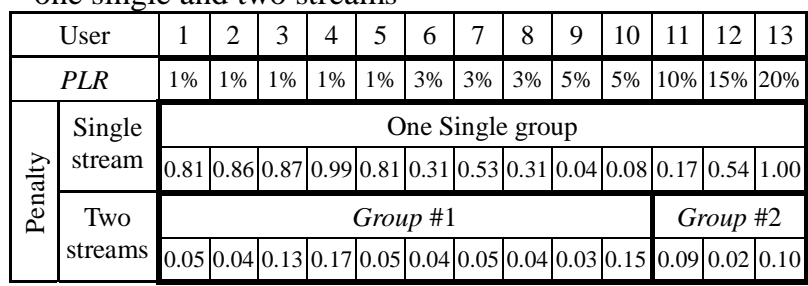

\section{CONCLUSION}

With the proposed CAIR-PT error resilience transcoder and fairness consideration, we proposed an efficient method to cope with more general video multicasting situations involving heterogeneous clients with diverse channel conditions. We have proposed a MINMAX loss rate estimation scheme to determine an appropriate intra-refresh rate for all the clients in a multicast group. We have also proposed a grouping method to partition a group of heterogeneous users into a minimal number of sub-groups to meet a given quality variation constraint while minimizing the channel bandwidth consumption under the quality constraint. Simulation results show that the proposed scheme can effectively reduce the mean and variance of penalty distortion of all users to achieve fairness.

\section{REFERENCES}

[1] J. Xin, C.-W. Lin, and M.-T. Sun, "Digital video transcoding,” Proc. IEEE, vol. 93, no. 1, pp. 84-97, Jan. 2005.

[2] J. Xin, A. Vetro, and H. Sun, "Error resilience video transcoding for wireless communications," IEEE Wireless Commun., no. 12, pp.14-21, Aug. 2005.

[3] S. Dogan, A. Cellatoglu, M. Uyguroglu, A. H. Sadka, and A. M. Kondoz, "Error-resilient video transcoding for robust internetwork communications using GPRS," IEEE Trans. Circuits Syst. Video Technol., vol. 12, no. 6, pp. 453-464, June 2002.

[4] H.-J. Chiou, Y.-R. Lee, C.-W. Lin, “Content-aware error resilient transcoding using prioritized intra-refresh for video streaming,” J. Visual Commun. Image Represent., vol. 16, pp. 563-588, Aug.-Oct. 2005.

[5] C.-M. Chen , C.-W. Lin, and Y.-C. Chen, "Error resilience transcoding using content-aware intra-refresh based on profit tracing," in Proc. IEEE Int. Symp. Circuits and Systems, May 2006, Island of Kos, Greece.

[6] R. Zhang, S. L. Regunathan, and K. Rose, "Video coding with optimal inter/intra-mode switching for packet loss resilience,” IEEE J. Select. Areas Communications, vol. 18, no. 6, pp. 966-976, June 2000.

[7] G. Cote and F. Kossentini, "Optimal intra coding of blocks for robust video communication over the internet," Signal Processing: Image Communication, vol. 15, pp. 25-34, Sept. 1999

[8] W.-H. J. Chen and J.-N. Hwang, "The CBERC: A contentbased error-resilient coding technique for packet video communications," IEEE Trans. Circuits Systems Video Technol., vol. 11, no. 8, pp. 974-980, Aug. 2001.

[9] S. Y. Cheung, M. H. Ammar, and X. Li, "On the use of destination set grouping to Improve fairness in multicast video distribution," in Proc. IEEE INFOCOM, Mar. March 1996, pp. 553-560.

[10] Z. Liu, H. Liu, and Y. Wang, "Cross layer adaptation for H.264 video multicasting over wireless LAN," in Proc. IEEE Int. Conf. Multimedia and Expo, July 2006, pp. 1121-1124.

[11] J. Youn, M.-T. Sun, and C.-W. Lin, "Adaptive motion vector refinement for high performance transcoding," IEEE Trans. Multimedia, vol. 1, no. 1, pp. 30-40, Oct. 1999.

[12] ISO/IEC 14496 (MPEG-4) Video Reference Software, Version: Microsoft-FDAM1-2.3-001213

[13] E. N. Gilbert, "Capacity of a burst-noisy channel,” Bell Syst. Tech. J., vol. 39, pp. 1253-1265, Sept. 1960. 